\title{
Functional outcomes according to the size of the gastric remnant and type of reconstruction following laparoscopic distal gastrectomy for gastric cancer
}

\author{
Eiji Nomura $\cdot$ Sang-Woong Lee $\cdot$ George Bouras $\cdot$ \\ Takaya Tokuhara $\cdot$ Michihiro Hayashi • \\ Masako Hiramatsu $\cdot$ Jyunji Okuda $\cdot$ Nobuhiko Tanigawa
}

Received: 7 September 2010/ Accepted: 7 March 2011/Published online: 26 April 2011

(C) The International Gastric Cancer Association and The Japanese Gastric Cancer Association 2011

\begin{abstract}
Background In gastric cancer, various methods of gastric resection and reconstruction have been devised according to the location of the primary tumor and the depth of invasion. The functional outcomes of patients treated by laparoscopy-assisted or totally laparoscopic distal gastrectomy were compared with respect to the approach, size of the remnant stomach, and type of reconstruction.

Methods Patients who required distal gastrectomy to treat early-stage cancer between May 2000 and December 2008 were treated by one of the four following procedures: Billroth Type I (B-1) reconstruction for $1 / 2$ remnant stomach $(1 / 2 \mathrm{~B} 1 \mathrm{ML})$ or $\mathrm{B}-1$ for $1 / 3$ remnant stomach (1/3B1ML), through a mini-laparotomy following laparoscopy-assisted surgery; intra-corporeal B-1 for $1 / 2$ remnant stomach (1/2 B1IC); or intra-corporeal Roux-en-Y for $1 / 3$ remnant stomach (1/3RYIC). The primary outcome measure was digestive function, assessed by body weight, food intake, and degree of abdominal symptoms. The secondary outcome was morbidity.

Results The 1/2B1ML $(n=27)$ and 1/2B1IC $(n=56)$ groups were significantly superior to the $1 / 3$ resection groups in terms of the preservation of body weight. The 1/3B1ML $(n=29)$ and 1/3RYIC $(n=64)$ groups were associated with significantly decreased food intake compared with the 1/2B1ML group. Endoscopy revealed a greater incidence of esophagitis and gastritis among the 1/3B1ML patients compared with the 1/3RYIC patients.
\end{abstract}

E. Nomura $(\bowtie) \cdot$ S.-W. Lee · G. Bouras · T. Tokuhara

M. Hayashi · M. Hiramatsu - J. Okuda - N. Tanigawa

Department of General and Gastroenterological Surgery,

Osaka Medical College, 2-7 Daigaku-Machi,

Takatsuki 569-8686, Japan

e-mail: sur035@poh.osaka-med.ac.jp
There were no operative deaths, and no differences in morbidity between the groups.

Conclusion Patients with early-stage cancer actually benefit from $1 / 2$ gastrectomy rather than the typical $2 / 3$ gastrectomy. B-1 reconstruction is appropriate for patients with large gastric remnants, and intra-corporeal reconstruction in experienced hands is associated with no apparent disadvantages, while offering a favorable cosmetic result.

Keywords Gastric cancer - Laparoscopic distal gastrectomy · Reconstruction - Surgical technique . Quality of life

\section{Introduction}

The incidence of early gastric cancer has increased in recent years [1]. As patients are expected to survive for longer after surgery, there has been increasing demand for less invasive and safe operative procedures which are associated with improved postoperative quality of life [2]. Consequently, various methods of laparoscopic resection and reconstruction have been devised according to the location of the primary tumor and the depth of invasion [3-5].

Standard-type distal gastrectomy for advanced cancer, as defined by the Japanese gastric cancer treatment guidelines [6] requires resection of $>2 / 3$ of the stomach. However, reduction of the extent of gastric resection is by all means possible for early-stage cancer, depending on the location of the tumor. Although a 1/2 gastrectomy may seem atypical for gastric cancer surgery, our previous experience with conventional open surgery suggests that a reduction in the extent of gastric resection is associated 
with improvement in the postoperative quality of life [7]. A 1/2 gastrectomy, therefore, is our standard when we perform laparoscopic gastrectomy, provided a sufficient resection margin can be obtained, while a typical $2 / 3$ gastrectomy is reserved essentially for cancer in the middle-third portion of the stomach. As for the reconstruction, a Billroth type I (B-1) procedure through a mini-laparotomy wound has been the most widely performed method upon the introduction of the laparoscopy-assisted approach to gastric cancer surgery [8]. More recently, a totally laparoscopic approach was reported as a feasible and potentially less invasive option. Thus, intra-corporeal stapled B-1 [9] is the current choice at our institution for a $1 / 2$ gastrectomy, while intra-corporeal Roux-en-Y reconstruction (RY) [10] is performed when the gastric remnant is smaller. In the present study, we retrospectively compared functional outcomes and morbidity between different types of reconstruction following laparoscopy-assisted and totally laparoscopic $1 / 2$ or $2 / 3$ distal gastrectomy for gastric cancer.

\section{Patients and methods}

One hundred and seventy-six consecutive patients who underwent laparoscopic distal gastrectomy between May 2000 and December 2008 at the Department of General and Gastroenterological Surgery, Osaka Medical College, were evaluated. To comply with the Japanese guidelines for the treatment of gastric cancer [6], only patients with a preoperative diagnosis of up to $\mathrm{T} 2$ stage with no markedly enlarged regional lymph nodes, as detected by computerized tomography, were considered to be eligible for the laparoscopic approach. Patients with a preoperative diagnosis of mucosal cancer of less than $4 \mathrm{~cm}$ in diameter or submucosal cancer of less than $2 \mathrm{~cm}$ in diameter located in the middle-third portion of the stomach were indicated for pylorus-preserving gastrectomy and were excluded from the study. Either $1 / 2$ or $2 / 3$ of the distal stomach was resected depending on the location of the tumor, and this was accompanied by dissection of perigastric lymph nodes up to $\mathrm{D} 1+\beta$ (dissection of lymph node stations No. 7, 8a, and 9 in addition to the perigastric nodes) [10]. The hepatic branch of the vagal nerve was routinely preserved, but preservation of the celiac branch was not considered. The size of the remnant stomach was determined as either $1 / 2$ or $1 / 3$ by the operating surgeon during surgery, following gastrectomy and scrutiny of the resected specimen and was recorded in the operative note. The clinicopathological findings of the gastric resections were recorded according to the Japanese classification of gastric carcinoma 2nd English edition [11].

\section{Outcome measures}

The primary outcome measure was the postoperative digestive function in terms of the postoperative/preoperative body weight ratio, postoperative/preoperative meal intake ratio, and the degree of postprandial abdominal symptoms. These data were acquired at one time point, 6-12 months postoperatively, through an in-house questionnaire (Table 1). In addition, the findings of patients who underwent endoscopy postoperatively at the outpatient clinic were analyzed to investigate the incidence of esophagitis and remnant gastritis. Endoscopic findings of the gastric remnant were evaluated according to the "residue, gastritis, bile" classification [12], and esophagitis was categorized by the Los Angeles classification. The incidences of gastritis, residue of $\geq$ Grade 2 , and esophagitis $\geq$ Grade A were considered as findings of clinical significance.

Data on postoperative complications, including anastomotic leakage, anastomotic stenosis, and gastric stasis were retrieved from the medical records as secondary outcome measures of the present study.

\section{Surgical procedures}

To determine whether to perform a $1 / 2$ or a $2 / 3$ gastrectomy, the resection line was, in principle, determined intraoperatively so as to ensure a $2-\mathrm{cm}$ margin from the macroscopic edge of the tumor. This was often made possible through preoperative endoscopic submucosal tattooing with $0.1 \mathrm{ml}$ India ink together with mucosal clipping. When in doubt, the

Table 1 Questionnaire survey about postoperative body weight, meal intake, and abdominal symptoms

1. Please state your body weight at present. __ $\mathrm{kg}$

2. Please put a circle around the number below that fits your present postoperative whole amount of meal intake per day compared to your preoperative whole meal intake.

1) $20 \%$

2) $40 \%$

3) $60 \%$

4) $80 \%$

5) $100 \%$

6) Other__ $\%$

3. Please put a circle around the number below that fits your description of abdominal symptoms that occur often, especially after meals, at present.

1) Borborygmus (stomach rumbling)

2) Abdominal pain

3) Diarrhea

4) Nausea or vomiting

5) Abdominal sensation of feeling full

6) Abdominal discomfort

7) Heartburn or reflux

8) No symptoms 
position of the clips was confirmed by X-ray or through a small gastrotomy to ensure that a sufficient resection margin was obtained. Resection and reconstruction were performed by one of the following 4 methods, depending on the location of the tumor and at the discretion of the surgeons: B-1 reconstruction through a mini-laparotomy wound for $1 / 2$ remnant stomach $(1 / 2 \mathrm{~B} 1 \mathrm{ML}), \mathrm{B}-1$ reconstruction through a mini-laparotomy wound for $1 / 2$ remnant stomach (1/3B1ML), intra-corporeal B-1 for $1 / 2$ remnant stomach (1/2B1IC), and intra-corporeal RY for $1 / 3$ remnant stomach (1/3RYIC). RY for large (1/2) remnant stomach had been performed before the study period but was soon abandoned due to an intolerable incidence of postoperative stasis. Intracorporeal stapled B-1 was performed as first reported by Kanaya et al. [9] in 2002. In brief, following resection, entry holes are made to the duodenum and the gastric remnant and a linear stapler is inserted to complete a side-to-side gastroduodenostomy. The remaining enterotomy is closed after approximating the bowel wall with stay sutures, using 2 further firings of the linear stapler. For intra-corporeal RY, we fashion a side-to-side anti-peristaltic gastrojejunostomy $30 \mathrm{~cm}$ from the ligament of Treitz, after which the jejunum is divided and the enterotomy is closed using a further firing of the linear stapler. The jejunojejunostomy is subsequently created extra-corporeally through an extended umbilical port wound, either by hand-sewing or using the stapler to complete the reconstruction [10].

\section{Statistics}

Statistical analysis was performed using Students $t$-test and the $\chi^{2}$ test. A $p$ value of less than 0.05 was considered to be significant.

\section{Results}

Of the 176 patients who underwent laparoscopic distal gastrectomy, 112 patients $(64 \%)$ completed the questionnaires on digestive functions. The patient demographics, stratified according to the surgical procedure, are presented in Table 2. No significant differences among the 4 groups undergoing different procedures were observed in age, sex, depth of invasion, clinical stage, the interval between surgery and the functional evaluation, and response rates for the questionnaires. There were fewer tumors located in the middle-third of the stomach among the $1 / 2 \mathrm{~B} 1 \mathrm{ML}$ group when compared with the $1 / 3 \mathrm{~B} 1 \mathrm{ML}$ and $1 / 3 \mathrm{R} Y \mathrm{C}$ groups. A $1 / 2$ gastrectomy was performed for $35 / 54$ (65\%) of patients with the lesion located in the lower-third of the stomach, whereas a $2 / 3$ gastrectomy was performed in 41/58 (71\%) of patients whose lesion was in the middle-third of the stomach. The 1/2B1IC was performed with a significantly shorter operating time and smaller blood loss compared with the other 3 procedures. There were no operative or inhospital deaths. The incidence of anastomotic leakage in the $1 / 2 \mathrm{~B} 1 \mathrm{ML}, 1 / 3 \mathrm{~B} 1 \mathrm{ML}, 1 / 2 \mathrm{~B} 1 \mathrm{IC}$, and $1 / 3 \mathrm{RYIC}$ groups was $11.1,3.4,0$, and $1.6 \%$, and that of anastomotic stricture was $3.7,3.4,1.8$, and $0 \%$, respectively. Gastric stasis occurred in $7.4,6.9,0$, and $4.7 \%$ of the $1 / 2 \mathrm{~B} 1 \mathrm{ML}$, 1/3B1ML, 1/2B1IC, and 1/3RYIC groups, respectively. Although not statistically significant, 1/2B1ML was associated with an increased incidence of anastomotic leakage. Overall, intra-corporeal reconstruction was not associated with higher complication rates compared to reconstruction through a mini-laparotomy.

Follow-up revealed that there was no evidence of recurrence at 1 year after surgery in any of the patients.

Table 2 Preoperative and operative characteristics of patients according to type of reconstruction

\begin{tabular}{lllll}
\hline & $\begin{array}{l}1 / 2 \mathrm{~B} 1 \mathrm{ML} \\
(n=17)\end{array}$ & $\begin{array}{l}1 / 3 \mathrm{~B} 1 \mathrm{ML} \\
(n=16)\end{array}$ & $\begin{array}{l}1 / 2 \mathrm{~B} 1 \mathrm{IC} \\
(n=35)\end{array}$ & $\begin{array}{l}1 / 3 \mathrm{RYIC} \\
(n=43)\end{array}$ \\
\hline Age (years) & $64.5 \pm 9.6$ & $60.9 \pm 14.3$ & $67.6 \pm 8.9$ & $65.9 \pm 10.5$ \\
Sex (male/female) & $12 / 5$ & $13 / 3$ & $21 / 14$ & $31 / 13$ \\
Location (M/L) & $2 / 15$ & $11 / 5^{\mathrm{a}}$ & $15 / 20$ & $30 / 14^{\mathrm{a}}$ \\
Depth (T1/T2) & $17 / 0$ & $15 / 1$ & $34 / 1$ & $41 / 3$ \\
Stage (IA/IB/II) & $17 / 0 / 0$ & $14 / 1 / 1$ & $33 / 2 / 0$ & $37 / 4 / 3$ \\
Operation time (min) & $281.2 \pm 78.3$ & $320.3 \pm 43.8$ & $250.4 \pm 59.2^{\mathrm{b}}$ & $350.0 \pm 75.6$ \\
Blood loss (ml) & $91.8 \pm 92.6$ & $70.6 \pm 66.3$ & $29.1 \pm 37.4^{\mathrm{b}}$ & $67.0 \pm 91.2$ \\
Interval (months) & $9.9 \pm 1.4$ & $9.8 \pm 1.4$ & $9.5 \pm 1.4$ & $9.4 \pm 1.6$ \\
Response rate (\%) & 63.0 & 55.2 & 62.5 & 67.2 \\
\hline
\end{tabular}

$L$ lower third of stomach, $1 / 2 B 1 M L$ B-1 reconstruction through a mini-laparotomy wound for $1 / 2$ remnant stomach, $1 / 3 B 1 M L$ B-1 reconstruction through a mini-laparotomy wound for $1 / 3$ remnant stomach, $1 / 2 B 1 I C$ intra-corporeal B-1 for $1 / 2$ remnant stomach, $1 / 3 R Y I C$ intra-corporeal RY for $1 / 3$ remnant stomach

${ }^{\text {a }}$ There were significantly fewer tumors in the middle third $(\mathrm{M})$ of the stomach for $1 / 2 \mathrm{~B} 1 \mathrm{ML}$ compared to $1 / 3 \mathrm{~B} 1 \mathrm{ML}$ and $1 / 3 \mathrm{RYIC}(p<0.05)$

${ }^{\mathrm{b}}$ Operation time and blood loss in the 1/2B1IC group was significantly lower than these parameters in the other 3 groups $(p<0.05)$

${ }^{c}$ Months between surgery and follow-up assessment 
Functional outcomes at 6-12 months

Comparison of the postoperative/preoperative body weight ratios (Fig. 1a) revealed better preservation of postoperative body weight among those with a larger remnant stomach (the $1 / 2 \mathrm{~B} 1 \mathrm{ML}$ and $1 / 2 \mathrm{~B} 1 \mathrm{IC}$ groups). The postoperative/preoperative food intake ratio was also significantly higher in the $1 / 2 \mathrm{~B} 1 \mathrm{ML}$ group than those in the 1/3B1ML and 1/3RYIC groups (Fig. 1b). Complaints of postprandial symptoms were significantly more common after the $1 / 3 \mathrm{~B} 1 \mathrm{ML}$ and $1 / 3 \mathrm{R} Y \mathrm{IC}$ procedures than after the 1/2B1IC procedure (Fig. 1c). Abdominal pain (24\%) and diarrhea $(18 \%)$ were frequent in the $1 / 3 \mathrm{~B} 1 \mathrm{ML}$ group, and abdominal pain $(20 \%)$ and nausea $(15 \%)$ were frequent in the 1/3RYIC group, while these symptoms were rarely observed in patients with a large gastric remnant. In summary, $1 / 2 \mathrm{~B} 1 \mathrm{ML}$ was significantly superior to the $1 / 3 \mathrm{~B} 1 \mathrm{ML}$ and $1 / 3$ RYIC procedures in terms of the amount of food intake ( $p=0.031$ and $p=0.013$, respectively), and it was significantly superior to the $1 / 3 \mathrm{R}$ YIC procedure in terms of preservation of body weight $(p=0.0002)$, while $1 / 2 \mathrm{~B} 1 \mathrm{IC}$ was superior to the $1 / 3 \mathrm{~B} 1 \mathrm{ML}$ and $1 / 3 \mathrm{RYIC}$ procedures regarding body weight $(p=0.017$ and $p \leq 0.0001$, respectively) and the incidence of abdominal symptoms ( $p=0.0003$ and $p=0.0034$, respectively). Thus, better functional outcomes were observed in patients with large gastric remnants, while no major differences were observed between B-1 and RY when the remnant stomach was small. Although not performed in all patients, endoscopy revealed that the incidence of reflux esophagitis in the $1 / 3 \mathrm{~B} 1 \mathrm{ML}$ group ( $n=14$ ) was significantly higher than that in the 1/3RYIC group ( $n=33$ ) (Fig. 2). Gastritis was observed in 2 of 14 patients in the $1 / 2 \mathrm{~B} 1 \mathrm{ML}$ group, 4 of 14 patients in the $1 / 3 \mathrm{~B} 1 \mathrm{ML}$ group, 4 of 30 patients in the $1 / 2 \mathrm{~B} 1 \mathrm{IC}$ group, and 3 of 33 patients in the 1/3RYIC group. Of these, $\geq$ Grade 2 residue was observed in $2,2,4$, and 3 patients, respectively.

\section{Discussion}

Since laparoscopic distal gastrectomy was first introduced in Japan, B-1 reconstruction has been the preferred method and it has been performed commonly either through a minilaparotomy [2] or intra-corporeally [9]. In addition to preservation of the duodenal passage, which may in theory be beneficial, B-1 has an advantage in that it can be completed with only one anastomosis [13]. Recently, RY has been adopted by several Japanese institutions to counter anastomotic leakage and duodenogastric reflux [14, 15]. In RY, the gastrojejunostomy can be performed without any tension even when the remnant stomach is small, and the anastomoses are distant from any potential a

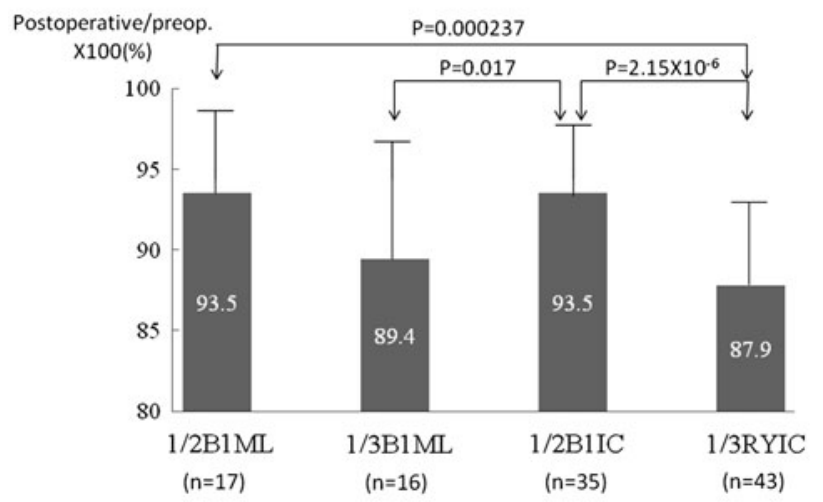

b

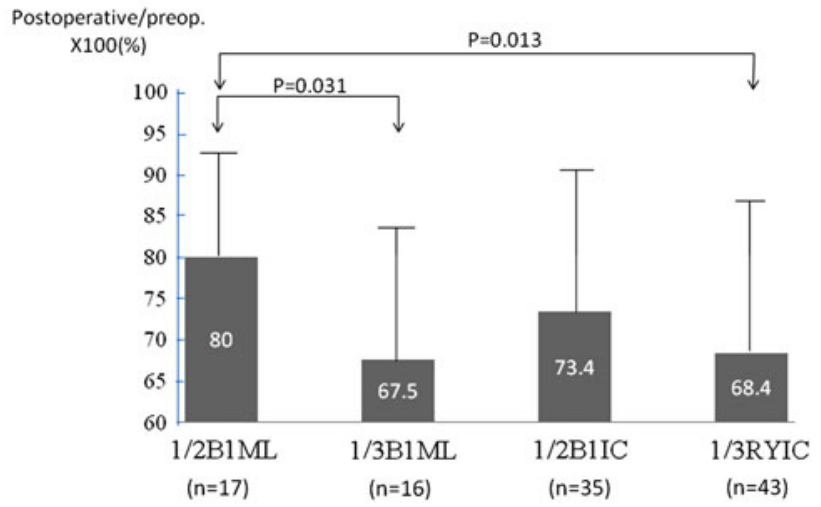

C

Postoperative/preop. $\mathrm{x} 100(\%)$

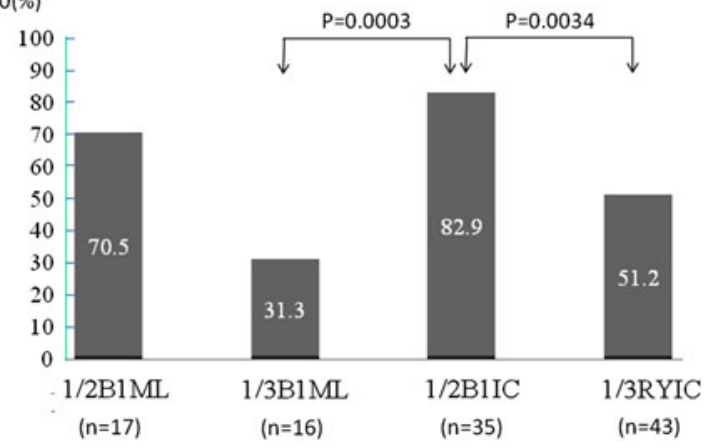

Fig. 1 Functional outcomes following laparoscopic distal gastrectomy according to type of reconstruction. a Postoperative/preoperative body weight ratios (\%). b Postoperative/preoperative meal intake ratios (\%). c Percentages of patients who had none of the abdominal symptoms listed in the questionnaire (Table 1). 1/2B1ML B-1 reconstruction through a mini-laparotomy wound for $1 / 2$ remnant stomach, $1 / 3 B 1 M L$ B-1 reconstruction through a mini-laparotomy wound for $1 / 3$ remnant stomach, $1 / 2$ B IIC intra-corporeal B-1 for $1 / 2$ remnant stomach, $1 / 3 R Y I C$ intra-corporeal RY for $1 / 3$ remnant stomach

pancreatic fistula-related collections that may arise from extensive lymph node dissection. In the light of the various types of reconstructive modalities available and through the evolution of our own surgical technique under the laparoscopic approach, we have achieved herein our 


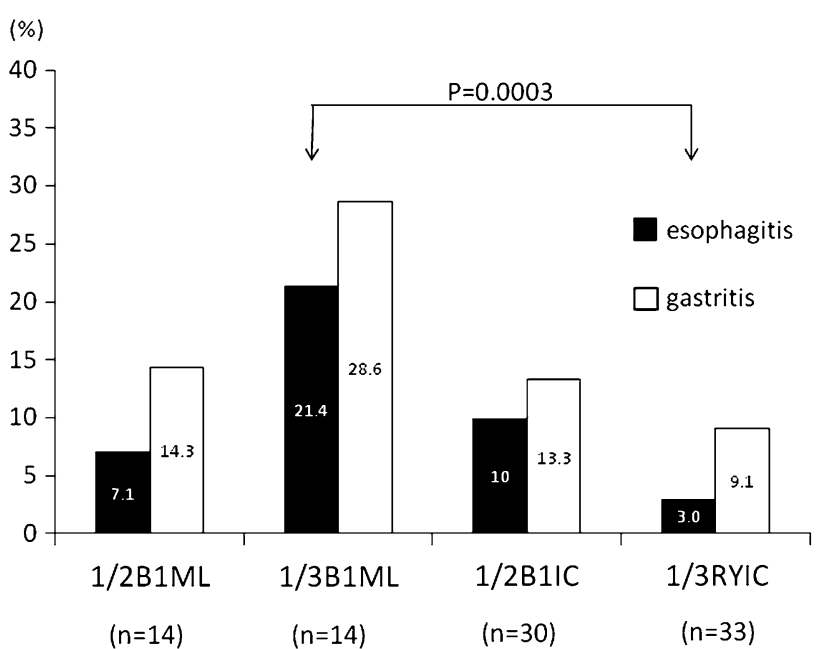

Fig. 2 The incidence of esophagitis and remnant gastritis

objectives of comparing functional outcomes according to the type of reconstruction, although this was done in a nonrandomized and retrospective fashion.

In terms of complications, the B1ML groups had the highest incidence of anastomotic leakage, although there were no statistically significant differences among the groups. This leakage may be related to the limited access for hand-sewn anastomosis through an epigastric minilaparotomy $4-6 \mathrm{~cm}$ in size. In contrast, intra-corporeal reconstruction offers a wide field of view with ample access and space to create the anastomosis. Furthermore, intra-corporeal B-1 was actually associated with significantly smaller blood loss compared to the other 3 groups. One could argue that the decrease in the incidence of anastomotic leakage and the smaller amount of blood loss may have reflected improvements in technical expertise in laparoscopic surgery rather than the benefit of the procedure per se. Our experience suggests, nevertheless, that the intra-corporeal anastomosis, which may, at a glance, seem complex is actually safe and technically feasible once sufficient experience with the laparoscopic surgery is attained. In addition, there is a potential for increased wound pain due to excessive stretching of the abdominal muscles during retraction. Tanimura et al. [16] suggested that totally laparoscopic gastrectomy was useful particularly for older patients with poor respiratory function.

In our previous experience, RY with a large remnant stomach was often associated with postoperative gastric stasis. The "RY syndrome", which reportedly occurs in about $30 \%$ of patients who undergo this mode of reconstruction [17] has been attributed to a disturbance of jejunal peristalsis [18]. This observation may be important when considering stasis from RY with large remnants, where gastric emptying occurs predominantly by gravity. When the remnant stomach is filled with food, it is apt to descend below the antecolic gastrojejunostomy, rendering gastric emptying through gravity difficult. Weakness in the peristalsis of the jejunum immediately aboral to the anastomosis would further hinder adequate passage of the food. Although a retrocolic gastrojejunostomy with the anastomosis fixed to the mesocolon may be useful to counter this problem, this is not an easy procedure to perform under the laparoscopic approach, particularly in patients with a thick mesocolon. On the other hand, Fujita et al. [15] analyzed 701 patients who underwent standard distal gastrectomy (defined as resection of more than $2 / 3$ of the stomach) with RY reconstruction by open surgery, and reported that delayed gastric emptying occurred only in 14 (1.9\%) patients. Our results also suggest that gastric emptying is unlikely to be a problem when the gastric remnant is small.

Fukuhara et al. [19] reported that the incidence of reflux symptoms such as epigastralgia, abdominal discomfort, and heartburn correlated well with the exposure of the gastric remnant to bile. Our results showing a decreased incidence of esophagitis in those reconstructed by RY agree with the increasing body of evidence that RY prevents bile reflux. In our previous analysis of open surgery for early gastric cancer, reduction of the extent of gastrectomy and preservation of the vagal branches and of the pyloric ring were associated with better quality of life [7]. The present data have shown that patients treated by the laparoscopic approach also benefit from a smaller $1 / 2$ resection. It can easily be speculated that the reservoir function of the remnant stomach is preserved in the $1 / 2$ resection group, leading to improved nutritional status and body composition. There is a potential weakness of the present study that internationally validated questionnaires were not used to evaluate the patient-reported outcomes. In this study, we evaluated the patients with the questionnaire format shown in Table 1 because the established instruments are not necessarily focused on specific postgastrectomy symptoms such as the dumping syndromes that we often encounter, and these instruments may not be sufficiently sensitive to detect subtle differences caused by small differences in the mode of reconstruction. In the present study, inconsistency in the intervals between surgery and the acquisition of functional data, ranging from 6 to 12 months, could also be criticized. It has been reported, however, that functional scores, symptom scales, and body composition tend to recover by 6 months after gastrectomy, with little difference thereafter, whereas these outcomes clearly reveal the worst values when evaluated at 1 month postoperatively $[20,21]$.

In conclusion, patients with early-stage cancer located in the lower-third of the stomach actually benefit from $1 / 2$ gastrectomy rather than the typical $2 / 3$ gastrectomy. B-1 reconstruction is appropriate for patients with a large gastric remnant, and intra-corporeal reconstruction in 
experienced hands is associated with no apparent disadvantages, while offering a favorable cosmetic result.

\section{References}

1. Maehara Y, Kakeji Y, Oda S, Takahashi I, Akazawa K, Sugimachi K. Time trends of surgical treatment and the prognosis for Japanese patients with gastric cancer. Br J Cancer. 2000;83: 986-91.

2. Kitano S, Shiraishi N, Fujii K, Yasuda K, Inomata M, Adachi Y. A randomized controlled trial comparing open vs laparoscopyassisted distal gastrectomy for the treatment of early gastric cancer: an interim report. Surgery. 2002;131:306-11.

3. Maki T, Shiratori T, Sugawa K. Pylorus-preserving gastrectomy as an improved operation for gastric ulcer. Surgery. 1967;61: 838-45.

4. Kameyama J, Ishida H, Yasaku Y, Suzuki A, Kuzu H, Tsukamoto M. Proximal gastrectomy reconstructed by interposition of a jejunal pouch. Eur J Surg. 1993;159:491-3.

5. Nomura E, Shinohara H, Mabuchi H, Lee SW, Sonoda T, Tanigawa N. Postoperative evaluation of the jejunal pouch reconstruction following proximal and distal gastrectomy for cancer. Hepatogastroenterology. 2004;51:1561-6.

6. The Japanese Gastric Cancer Association. Guidelines for the treatment of gastric cancer. Tokyo: Kanehara-Shuppan; 2001.

7. Nomura E, Isozaki H, Fujii K, Toyoda M, Niki M, Sako S, et al. Postoperative evaluation of function-preserving gastrectomy for early gastric cancer. Hepatogastroenterology. 2003;50:2246-50.

8. Kitano S, Iso Y, Moriyama M, Sugimachi K. Laparoscopyassisted Billroth 1 gastrectomy. Surg Laparosc Endosc. 1994;4: $146-8$.

9. Kanaya S, Gomi T, Momoi H, Tamaki N, Isobe H, Katayama T, et al. Delta-shaped anastomosis in totally laparoscopic Billroth 1 gastrectomy: new technique of intraabdominal gastroduodenostomy. J Am Coll Surg. 2002;195:284-7.
10. Takaori K, Nomura E, Mabuchi H, Lee SW, Agui T, Miyamoto $\mathrm{Y}$, et al. A secure technique of intracorporeal Roux-Y reconstruction after laparoscopic distal gastrectomy. Am J Surg. 2005;189:178-83.

11. Japanese Gastric Cancer Association. Japanese classification of gastric carcinoma. 2nd English edition. Gastric Cancer 1998;1: $10-24$.

12. Kubo M, Sasako M, Gotoda T, Ono H, Fujishiro M, Saito D, et al. Endoscopic evaluation of the remnant stomach after gastrectomy: proposal for a new classification. Gastric Cancer. 2002;5:83-9.

13. Kim BJ, O’Connell T. Gastroduodenostomy after gastric resection for cancer. Am Surg. 1999;65:905-7.

14. Nunobe S, Okaro A, Sasako M, Saka M, Fukagawa T, Katai H, et al. Billroth1 versus Roux-en-Y reconstructions: a quality-of life survey at 5 years. Int J Clin Oncol. 2007;12:433-9.

15. Fujita T, Katai H, Morita S, Saka M, Fukagawa T, Sano T. Shortterm outcomes of Roux-en-Y stapled anastomosis after distal gastrectomy for gastric adenocarcinomna. J Gastrointest Surg. 2010;14:289-94.

16. Tanimura S, Higashino M, Fukunaga Y, Kishida S, Ogata A, Fujiwara Y, et al. Respiratory function after laparoscopic distal gastrectomy - an index of minimally invasive surgery. World $\mathbf{J}$ Surg. 2006;30:1211-5.

17. Tu BN, Kelly KA. Motility disorders after Roux-en-Y gastrojejunostomy. Obes Surg. 1994;4:219-26.

18. Mathias JR, Fernandez A, Sninsky CA, Clench MH, Davis RH. Nausea, vomiting, and abdominal pain after Roux-en-Y anastomosis: motility of the jejunal limb. Gastroenterology. 1985;88: 101-7.

19. Fukuhara K, Osugi H, Takada N, Takemura M, Higashino M, Kinoshita $\mathrm{H}$. Reconstructive procedure after distal gastrectomy for gastric cancer that best prevents duodenogastroesophageal reflux. World J Surg. 2002;26:1452-7.

20. Kobayashi D, Kodera Y, Fujiwara M, Koike G, Nakayama G, Nakao A. Assessment of quality of life after gastrectomy using EORTC QLQ-C30 and STO22. World J Surg. 2011;35:257-62.

21. Abdiev S, Kodera Y, Fujiwara M, Koike M, Nakayama G, Ohashi $\mathrm{N}$, et al. Nutritional recovery after open and laparoscopic gastrectomies. Gastric Cancer. 2011 (in press). 